\title{
Identifying the weak spots in packaging paper: local variations in grammage, fiber orientation and density and the resulting local strain and failure under load
}

\author{
Jussi Lahti • Michael Dauer $・$ D. Steven Keller • Ulrich Hirn $\mathbb{1}$
}

Received: 5 May 2020/ Accepted: 24 September 2020/Published online: 6 October 2020

(C) The Author(s) 2020, corrected publication 2020

\begin{abstract}
Measured local paper structure-i.e. local basis weight, local thickness, local density and local fiber orientation-has been linked to local strain and local material failure (local temperature increase due to energy dissipation upon fiber-fiber bond failure) measured during tensile testing. The data has been spatially linked through data map registration delivering several thousand $1 \times 1 \mathrm{~mm}^{2}$ paper regions, each containing all measured properties. The relation between local paper structure and resulting local deformation and failure is studied with regression models. Multiple linear regression modeling was used to identify the paper structure related drivers for local concentrations of strain under load and local concentrations of material failure, which are both starting to
\end{abstract}

Electronic supplementary material The online version of this article (https://doi.org/10.1007/s10570-020-03493-z) contains supplementary material, which is available to authorized users.

J. Lahti · M. Dauer · U. Hirn ( $\square)$

Institute of Bioproducts and Paper Technology, Graz

University of Technology, Graz, Austria

e-mail: ulrich.hirn@tugraz.at

J. Lahti · M. Dauer · U. Hirn

Christian Doppler Laboratory for Fiber Swelling and

Paper Performance, Vienna, Austria

D. S. Keller

Department of Chemical, Paper and Biomedical

Engineering, Miami University, Oxford,

$\mathrm{OH}, \mathrm{USA}$ occur considerably before rupture of the paper. Analyzing the development of local strain in paper we found that regions with higher basis weight and higher fiber orientation in load direction tend to exhibit considerably lower strain during tensile testing. Furthermore, the relation between local strain and local grammage can be predicted with the statistical theory of elasticity. Also regions with higher density have lower local strain, but not as pronounced. The findings for local fiber-fiber bond failure of paper are similar but not equivalent. The strongest correlation exists with local grammage. Local density and local fiber orientation show in turn weaker correlation with local bond failure. Local variations in paper thickness were not relevant in any case. These findings are highlighting the relevance of local fiber orientation and local density variations as structural mechanisms governing paper failure. In the past the focus has been mostly on paper formation. Together with local grammage (formation) they are responsible for the weak spots in paper, and thus cause local concentrations of paper strain and the initiation of failure under tensile load.

Keywords Natural fibres $\cdot$ Cellulose $\cdot$ Stress concentrations · Damage mechanics

\section{Introduction}

Paper is produced from a pulp suspension including fibers, fiber segments (fines) and chemical additives. 
During forming process these components settle stochastically in the wet paper web formed on the wire. This in combination with subsequent process steps, i.e. wet pressing and drying, leads to a heterogeneous paper structure with locally varying properties such as basis weight, fiber orientation, density, internal stresses due to drying shrinkage/wet strain, interfiber bonding degree and fines content (Korteoja et al. 1996; Alava and Niskanen 2008; Niskanen and Pakarinen 2008). It has been discussed that all these structural non-uniformities combined lead to an uneven strain and damage distributions within paper under in-plane tensile load which in turn reduces the papers ability to globally withstand tensile stresses without breaking. The structural non-uniformities are creating weak spots with low stiffness in the paper where the high strains are localized under load. These highly strained weak spots act as precursors for material failure (Norman 1965; Korteoja et al. 1996, 1997, 1998; Alava and Niskanen 2008; Hagman and Nygårds 2012; Borodulina et al. 2012; Romanova et al. 2020). A particularly interesting question is which structural inhomogeneities are responsible for the weak spots causing high local strains and the onset of paper failure under load.

Previous experimental studies in the field of paper have focused only on the relationships between local basis weight, local tensile strain and local tensile damage. In the seminal work of Dodson (1970) a significant correlation between local deformation and local basis weight was found. The local deformation was investigated microscopically and the local basis weight by gravimetrically measuring the local regions of fibrous networks. Korteoja et al. (1998) as well as Hagman and Nygårds (2017) concluded that locally high tensile strain and tensile damage occurred in the regions with low basis weight. Besides, Wong et al. (1996) found that the rupture of paper initiated from the region with low basis weight and high strain. Yamauchi and Murakami (1994) in turn stated that the basis weight was low and the temperature increase high in the initiation region of the rupture. The local strain was measured with digital image correlation (DIC) in each case. Korteoja et al. (1998) studied local tensile damage optically with the aid of silicon impregnation which improved the visibility of the damaged regions. Hagman and Nygårds (2017) as well as Yamauchi and Murakami (1994) utilized in turn infrared thermography (IRT) to investigate the damage distribution. This method is based on the fact that thermal energy is dissipated (temperature increases) in the regions with plastic deformation, i.e. breaking of interfiber bonds, plastic deformation of fibers and friction between moving fibers. Korteoja et al. (1998) and Wong et al. (1996) measured local basis weight with a $\beta$-radiographic transmission method (described in "Local basis weight" section). Yamauchi and Murakami (1994) utilized a soft X-ray method and Hagman and Nygårds (2017) optical method to estimate the basis weight variations within paper.

Simultaneous local tensile strain and local tensile damage measurements in combination with structural analysis has been conducted also for other types of composites. Goidescu et al. (2013) studied fiberreinforced polymer (FRP) by measuring local tensile strain and local tensile damage with DIC and IRT. For investigating the damage types within the deformed regions, e.g. whether the damage occurred through breakage of fibers or fiber-matrix debonding, they used X-ray tomography. Similar kind of methodology was developed by Battams and Dulieu-Barton (2016). They conducted DIC and thermoelastic stress analysis (TSA) during a fatigue test of FRP in a synchronized manner. The damage types were verified with X-ray tomography also in this case.

The aim of the current study is to advance the understanding of distribution of strain and damage to a quantitative analysis of the structural reasons for paper failure. There are two novel aspects in the current work. First, earlier studies on paper have focused only on the relationships between local basis weight, local strain and local damage. In order to better understand the structural mechanisms governing local strain and local damage in a more comprehensive manner, we have added three additional local structural properties, those are local thickness, local density and local fiber orientation (FO). Two of these properties, fiber orientation and density, are known to have a strong impact on paper stiffness and strength, similarly to basis weight, on a global sheet level (I'Anson et al. 2008; Alava and Niskanen 2008). The tensile stiffness index and tensile index of low basis weight papers increase with increasing basis weight. At higher basis weights, the tensile stiffness index and tensile index typically start to decrease. However, this decrease does not occur for denser papers (I'Anson et al. 2008). As basis weight influences both locally and globally 
on the mechanical properties of paper, it can be expected that the fiber orientation and density do so as well. Second we are providing a quantitative investigation of the relations between paper strain/failure under load and paper structure. This is achieved by linking the local structural properties to the local strain/failure of the paper under load using image registration of the respective paper property maps (Hirn et al. 2008). The paper is divided into $1 \times$ $1 \mathrm{~mm}^{2}$ subregions and all the structural and deformation properties are measured for each of these subregions. Using the large amount of local datapoints permits quantitative analysis of the local failure/ deformation behavior as a function of the local paper structural properties using linear regression models.

\section{Materials and methods}

\section{Principle}

In this study the local structural properties of four $73 \mathrm{~g} / \mathrm{m}^{2}$ Kraft paper strips are linked to their strain and damage distributions during tensile testing. The paper is produced from unbleached softwood Kraft pulp. Figure 1 illustrates the principle to link local paper properties by showing four maps of the same paper specimen imaged in different ways: (a) a photograph, (b) an IR thermogram, (c) a $\beta$-radiograph. In the last analysis step each sample is split into 14-18 layers to measure the local fiber orientation, Fig. 1d shows a high resolution image of one of the split layers, the enlarged region shows that the local fiber orientation is visible. The dashed lines are indicating the region of interest (ROI) where all local paper parameters were determined in a resolution of $1 \mathrm{~mm}^{2}$.

The strips had dimensions of $40 \times 65 \mathrm{~mm}^{2}$ including a region of interest (ROI), i.e. the region where the linking of local properties was conducted, with dimensions of $35 \times 60 \mathrm{~mm}^{2}$. The ROI was marked identically on both sides of each specimen with four dots indicating the corners of the ROI. On the front side, where the strain map was determined with a photography during tensile testing and a subsequent DIC analysis, the marking was done with a felt pen. Furthermore, a random speckle pattern was printed (HP Color LaserJet 4700n) on the front side to enable an accurate DIC analysis. All relevant DIC hard- and software parameters are given in Online Resource 1. On the back side, where the IR thermogram was measured with an IRT during tensile testing simultaneously with the strain map, the landmark dots were marked with silver ink due to its high IR reflectance which made the dots clearly visible in the recorded thermograms. High local basis weight within the silver ink dots made them also clearly detectable in the measured $\beta$-radiographs. (a)

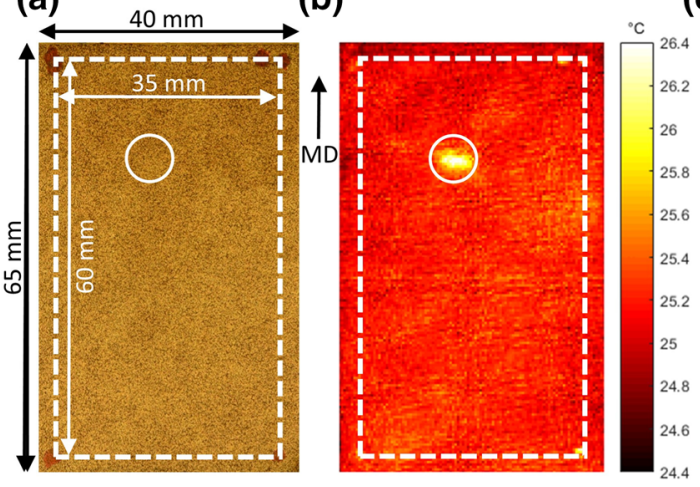

Fig. 1 Principle for linking local structural properties of a paper specimen to local strain and local damage during tensile testing. The figure shows four different property maps of the same paper specimen. a Optical image of specimen with random speckle pattern for local strain measurement with DIC, b IR thermogram of sample during tensile testing indicating local damage (energy dissipation due to locally concentrated failure of fiber bonds) $\mathbf{c}$ (c)

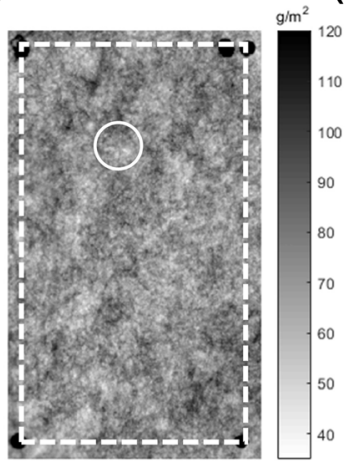

(d)

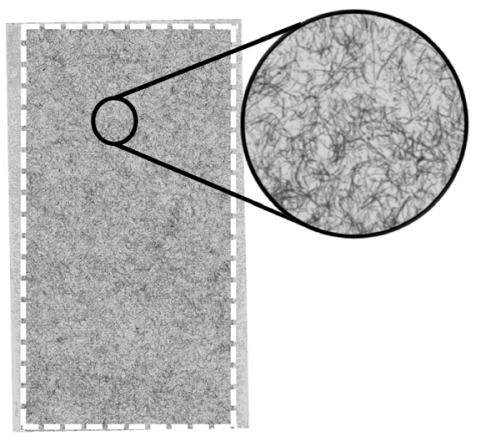

local grammage of sample measured with $\beta$-radiography and d optical image of one layer of the sample after sheet splitting for measurement of local fiber orientation. The rectangular region of interest (dashed line) can be identified in all four maps. The circle indicates a region with damage accumulation (high temperature in IR image) during tensile testing 
In addition, the region where considerable damage occurred just prior to tensile rupture is highlighted with a circle in the IR thermogram in Fig. 1. The same region is also marked to the photograph, the $\beta$ radiograph and the split layer of the specimen. This particular example illustrates that the damage occurred in the region with low basis weight surrounded by regions with high basis weights in the straining direction, i.e. machine direction (MD). In order to study the relationships between local structure, local strain and local damage comprehensively, local basis weight, local thickness, local density and local FO were linked to local strain and local temperature increase (thermal energy dissipation) just prior to tensile rupture as shown in Fig. 2. Local basis weight was studied with a $\beta$-radiographic transmission method, local thickness with a twin laser profilometer (TLP) and local density by dividing the former by the latter. A sheet splitting method was in turn utilized to measure the local fiber orientation (FO). As already mentioned, local temperature increase (thermal energy dissipation) was measured with infrared thermography IRT and local strain with the combination of photography during tensile testing and digital image correlation DIC. In the following all these measurement methods as well as the linking procedure are presented more in detail.

\section{Strain and damage distribution}

The set-up for measuring strain and damage distributions is shown in Fig. 3. The strain map was determined on the front side of the tensile tester (Zwick/Roell Z010) with a $10 \mathrm{kN}$ load cell. First the tensile test of the paper strip was recorded with a digital single-lens reflex (SLR) camera (Nikon D5100) combined with a macro lens (Nikon ED AF Micro Nikkor $200 \mathrm{~mm} \mathrm{1:4} \mathrm{D).} \mathrm{The} \mathrm{frame} \mathrm{rate} \mathrm{and} \mathrm{resolution}$

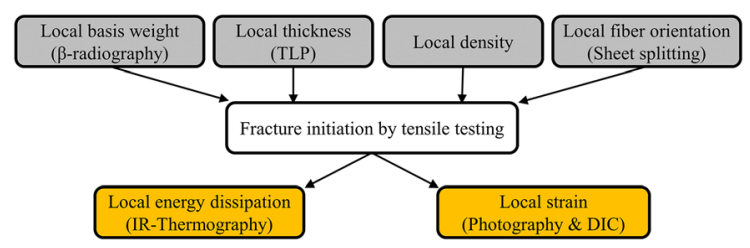

Fig. 2 Measurements for linking local structural properties (top row, compare Fig. 5a-d) of a paper specimen to its local strain and damage distributions (bottom row, compare Fig. 5e, f) during tensile testing of the recording were $25 \mathrm{~Hz}$ and $37 \mu \mathrm{m} /$ pixel, respectively. The ROI within recorded photographs was then analyzed with a two-dimensional (2D) DIC (Eberl et al. 2006) by utilizing the printed random speckle pattern. A comprehensive review of the 2D DIC is given by Pan et al. (2009). The DIC analysis was conducted with a subset size of $20 \times 20$ pixels and thus the strain map resolution became $0.74 \mathrm{~mm} / \mathrm{pixel}$. The step size was in turn 15 pixels $(0.56 \mathrm{~mm})$. Finally, the determined strain map was resized (MATLAB imresize function) to the size of the ROI just prior to rupture, i.e. $35 \times 61 \mathrm{~mm}^{2}$.

Simultaneously with the measurement of the strain map on the frontside, temperature increase (thermal energy dissipation) map was recorded on the backside of the tensile tester. The principle of studying paper damage with IRT is described in detail by Yamauchi (2012). The key idea is that during tensile testing fiber-fiber bonds are starting to fail long before the actual macroscopic rupture of the paper. The strain energy stored in the fibers is dissipated as heat when the bond between fibers is failing. Thus the local heat distribution in the paper provides information on where fiber-fiber bonds in paper are failing. Thus we use the local temperature as an indicator for local failure of the paper or local damage in the bonding of the paper.

We used a long-wavelength $(7.5-13 \mu \mathrm{m})$ IR camera (Optris PI 450). The long-wavelength IR emittance was approximated to be 0.93 (Hyll 2012). The frame rate and resolution of the recording were in turn $80 \mathrm{~Hz}$ and $0.38 \mathrm{~mm} /$ pixel, respectively. The temperature increase map just prior to tensile rupture was determined from the recorded thermograms by resizing (MATLAB imresize function) the ROI within thermogram prior to straining $\left(35 \times 60 \mathrm{~mm}^{2}\right)$ to the same size as the ROI within thermogram just prior to rupture $\left(35 \times 61 \mathrm{~mm}^{2}\right)$ and then subtracting the unstrained ROI from the strained one.

The tensile test was performed in MD with a strain rate of $40 \mathrm{~mm} / \mathrm{min}$ under a standard climate of $23^{\circ} \mathrm{C}$ and $50 \%$ relative humidity. The tensile test was not performed until complete rupture of the strip but stopped just prior to/during rupture, i.e. at the strain level of $2.2 \%$ (size of the ROI $35 \times 61 \mathrm{~mm}^{2}$ ). The reason was because the measurement of local fiber orientation (sheet splitting method) is also a destructive method, and thus the local structural properties 


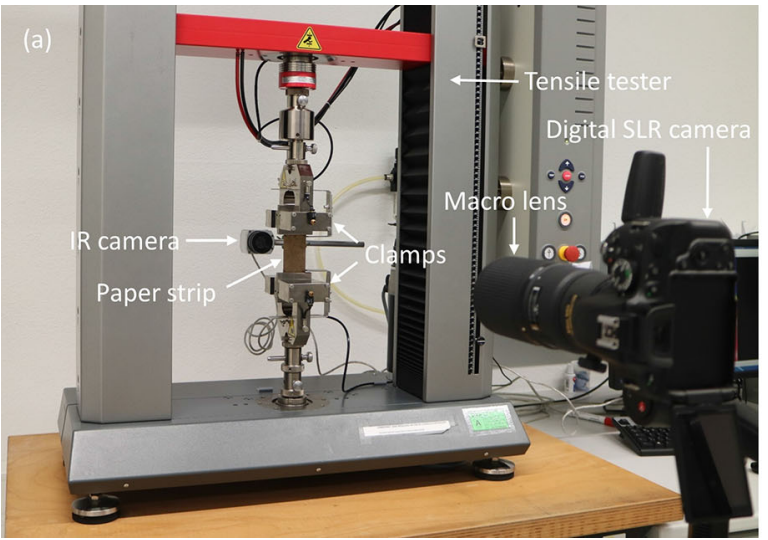

Fig. 3 Set-up for recording both local strain (photography in combination with DIC) and local temperature increase (IR thermography) simultaneously during tensile testing. a frontside

were measured after the measurement of strain and damage distribution.

The development of strain and temperature maps during tensile test was investigated through calculation of mean and variance over ROI (see Fig. 1). Every recorded strain map $(25 \mathrm{~Hz})$ and every fifth temperature map $(16 \mathrm{~Hz})$ was analyzed. For strain and temperature mean and variance were calculated.

\section{Local basis weight}

The local basis weight was measured with a $\beta$ radiographic transmission method described in detail by Keller and Pawlak (2001). First the $\beta$-radiographic transmission through a paper strip and references with known basis weights was recorded on a storage phosphor screen in a Formex Betaformation Box (Science Imaging Scandinavia AB). Second the screen was scanned (Fujifilm BAS 1800-II) and the basis weight map within the ROI of the strip was calculated with a resolution of $50 \mu \mathrm{m} /$ pixel by using the references.

\section{Local thickness and density}

The local thickness was measured with a TLP described in detail by Keller et al. (2012). TLP determined the surface topography of the paper strip simultaneously on both sides with the aid of two lasers. The local thickness was then calculated with a resolution of $100 \mu \mathrm{m} /$ pixel by using the surface
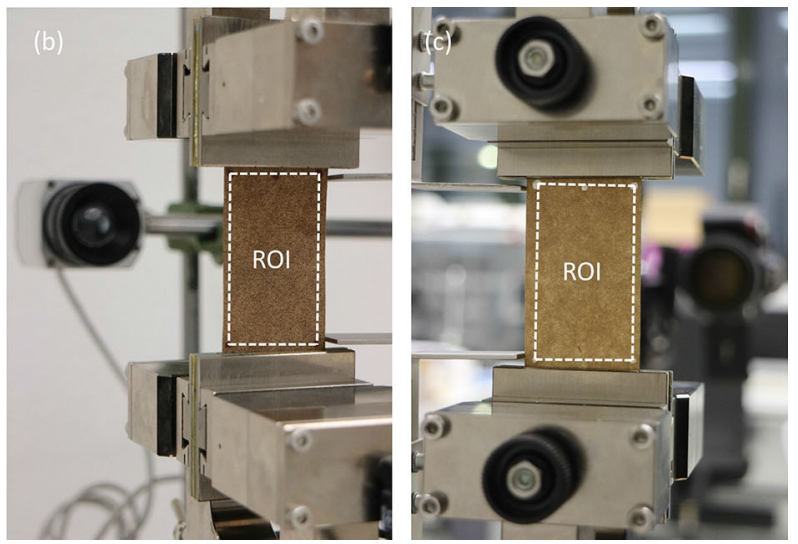

view of the set-up, b close-up view from the frontside where strain map is measured and c close-up view from the backside where temperature increase map is recorded

topographies. Due to the high local thickness within the silver ink dots, the dots were clearly visible in the thickness maps. Local density was calculated by dividing local grammage by local thickness.

\section{Local fiber orientation}

The local FO was measured with a sheet splitting method described in detail by Hirn and Bauer (2007). As it is a destructive method, the determination of local FO was the last measurement step. Before starting the procedure the landmark dots were made visible by bunching holes on the corners of the ROI. The local FO measurement was started by splitting the paper strip in thickness direction (ZD) into thin layers and subsequently scanning (Nikon Super CoolScan $8000 \mathrm{ED})$ the layers with a resolution of $13 \mu \mathrm{m} /$ pixel. Each scanned layer was then analyzed through image analysis. In other words, each scanned layer was divided into subsets of $1 \times 1 \mathrm{~mm}^{2}$ followed by a determination of FO distribution within each subset. The FO map for all the layers in local $1 \times 1 \mathrm{~mm}^{2}$ subsets was then determined by averaging the FO distributions of the layers in ZD and subsequently fitting orientation ellipses to the averaged distributions. The averaged FO distribution and fitted orientation ellipse of one subset are shown in Fig. 4c. The FO of a subset, i.e. FO vector, is defined with the aid of FO anisotropy and FO angle $(\theta)$. FO anisotropy is the eccentricity $(e)$ of the orientation ellipse calculated as follows: 


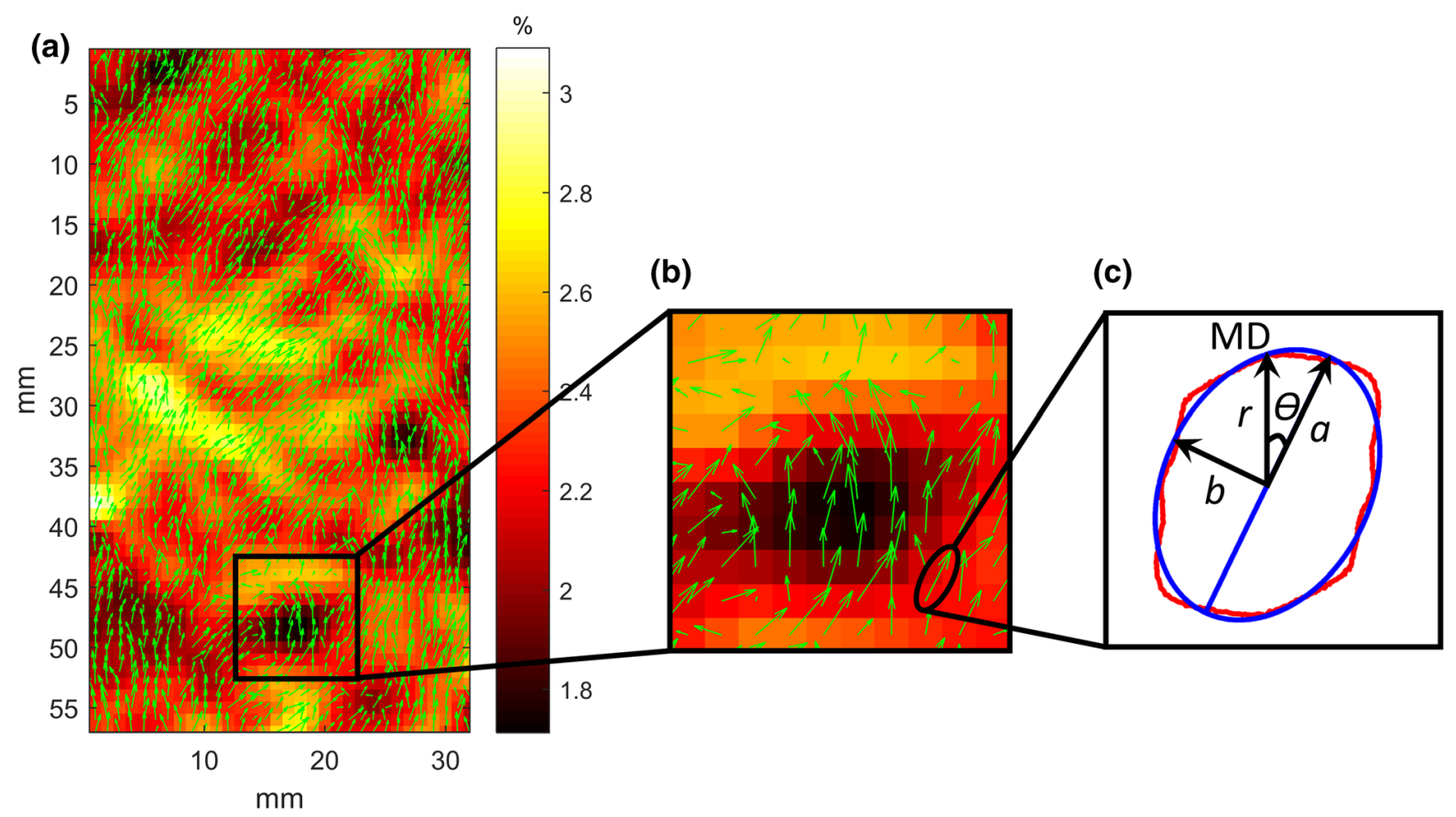

Fig. 4 Local fiber orientation is determined by accumulating the local fiber orientation in different layers after sheet splitting, see Fig. 1d. a FO map aligned on top of the corresponding strain map, b region including subsets with both high and low strain values and the corresponding FO vectors and $\mathbf{c}$ FO distribution (red) and fitted orientation ellipse (blue) for one $1 \times 1 \mathrm{~mm}^{2}$

$e=\sqrt{1-\frac{b^{2}}{a^{2}}}$

where $a$ is the major semi-axis and $b$ the minor semiaxis of the ellipse. FO angle $\theta$ is in turn the angle between the major semi-axis of the ellipse and MD. The magnitude and angle of the FO vector are then defined as the eccentricity and FO angle, respectively.

As local FO angle and local FO anisotropy combined are needed to investigate the influence of local FO on local strain/damage, a variable called FO load carrying factor (FOLCF) is introduced. It links the local FO to the local ability of the fiber network to carry tensile load and is technically defined as the local probability density of fiber segments pointing in load direction $(2 r)$ within a subset. The FOLCF is calculated by using the polar equation of the orientation ellipse as follows:

$$
F O L C F=2 r=\frac{2 a b}{\sqrt{(b \cos \theta)^{2}+(a \sin \theta)^{2}}}
$$

subregion. Magnitude and direction of each FO vector are defined as eccentricity e and angle $(\theta)$ of the orientation ellipse, respectively. FOLCF (fiber orientation load carrying factor) is technically defined as the local probability density of fiber segments pointing in load direction (vertical, vector $\mathrm{r}$ )

\section{Linking procedure}

In order to enable a quantitative pointwise correlation, the measured maps were aligned and rescaled to a resolution of $1 \times 1 \mathrm{~mm}^{2}$ per pixel. This was done through a landmark based image registration procedure by utilizing the four landmark dots visible in the corners of each map and aligning the images using these landmarks. The maps were aligned using a shape preserving coordinate transform, this procedure is described in Hirn et al. (2008). At this point the density map was also calculated by dividing the aligned basis weight map with the aligned thickness map. The noise of the aligned maps was then reduced by the aid of Gaussian low pass filter with a kernel size of $5 \times 5 \mathrm{~mm}$ pixels and a standard deviation of 1.0 (MATLAB imgaussfilt function).

The aligned dataset of one paper specimen is shown in Fig. 5. Please note that, as a result of the registration procedure described above, all the maps have a common coordinate system where each pixel represents a $1 \times 1 \mathrm{~mm}^{2}$ subarea on the paper specimen. 

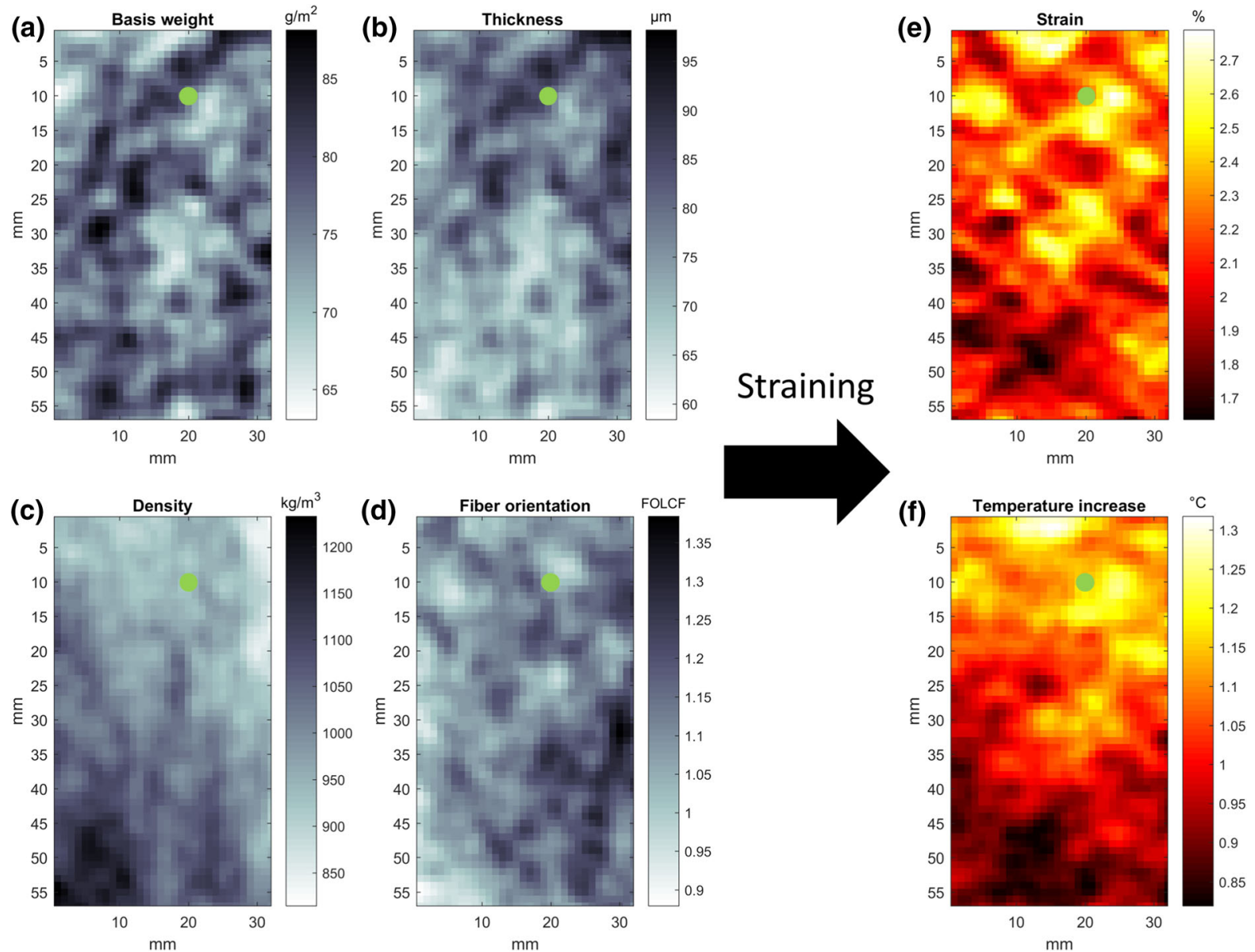

Fig. 5 Measured local paper structural properties (a-d), and the corresponding local strain (e) and local damage (f) during tensile testing. The maps all show a region of $32 \times 57 \mathrm{~mm}^{2}$ inside ROI (dashed rectangle in Fig. 1) of one paper specimen. The size of the final data maps was slightly smaller than ROI due to exclusion of the landmark dots from the analysis. Local maps of

Furthermore, the size of the final data maps was $32 \times 57 \mathrm{~mm}^{2}$, i.e. slightly smaller than the actual ROI just prior to rupture $\left(35 \times 61 \mathrm{~mm}^{2}\right)$, due to removal of the landmark dots from the analysis. By extracting all the structural parameters, the strain and the temperature from all the data maps at one specific coordinate position, all the local properties of a specific subarea on the sample can be collected. In this way 7296 data points with local structural and local deformation/damage data are collected from the four paper specimen analyzed. One of these data points at the coordinate position $\mathrm{x}=20 / \mathrm{y}=10$ is indicated in a basis weight, b thickness, c density, d fiber orientation (FOLCF, compare Fig. 4), e strain and $\mathbf{f}$ damage (IR thermography). Local properties are plotted in $1 \times 1 \mathrm{~mm}^{2}$ subregions, the green spot in each map indicates the local paper properties and resulting local deformation/damage at the position $\mathrm{x}=$ $20 / y=10$ on the sample

Fig. 5, its according properties can be read from the data maps.

In total 48 local data values were detected as outliers using Grubbs' test with a significance level of 0.05. The quantitative analysis of relations between strain map, damage map and structural maps was conducted through correlation, simple/multiple linear regression and analysis of variance (ANOVA) of the 7296 data points (outlier data values excluded) collected (Neter et al. 1996). 


\section{Results and discussion}

Global mean properties of the investigated Kraft paper

The investigated Kraft paper had a basis weight of $73 \mathrm{~g} / \mathrm{m}^{2}$ and density of $1041 \mathrm{~kg} / \mathrm{m}^{3}$. The global mean FO paramaters were in turn as follows: FO anisotropy 0.71 , FO angle $22.7^{\circ}$ and FOLCF 1.13. The tensile stress at break was $111 \pm 3 \mathrm{MPa}$ in $\mathrm{MD}$ and $58 \pm 2 \mathrm{MPa}$ in CD. However, in the current study the tensile test in MD was stopped just prior to rupture at strain level of $2.2 \%$ and the corresponding tensile stress was $100 \pm 2 \mathrm{MPa}$ (see Fig. 6a). In the following the local properties of the investigated Kraft paper are discussed.
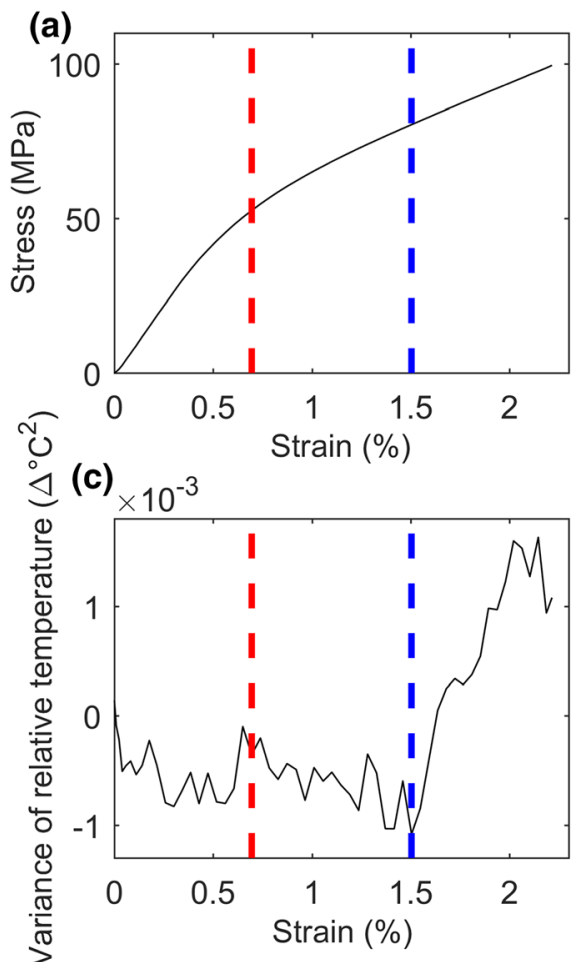

Fig. 6 Development of mean and variance of strain and temperature maps during tensile test for all four strips. Averaged a stress, b temperature, c variance of local temperature and d variance of strain as a function of averaged strain. The red line at $0.7 \%$ strain shows the onset of bond failure, which is indicated by an increase in temperature (b) caused by thermal dissipation of strain energy in failing fiber-fiber bonds. The blue line at
Qualitative analysis of local properties

Figure 5 qualitatively demonstrates the relationships between the measured local structural properties, the measured local deformation and the measured local damage. It shows the local basis weight, thickness, density, fiber orientation (FOLCF), strain and temperature increase for one of the studied paper strips. Clear mesostructural inhomogeneities are visible and similar conclusions can be made as in the previous investigations described in "Introduction" section of this paper. In other words, both the local strain and local temperature seem to correlate to a certain extent with the local basis weight. Similarities can be also seen between the strain and the damage distribution maps. Furthermore, the other structural maps seem to correlate to a certain extent with the strain and the damage distribution maps as well. In order to investigate these causalities in a more detailed and
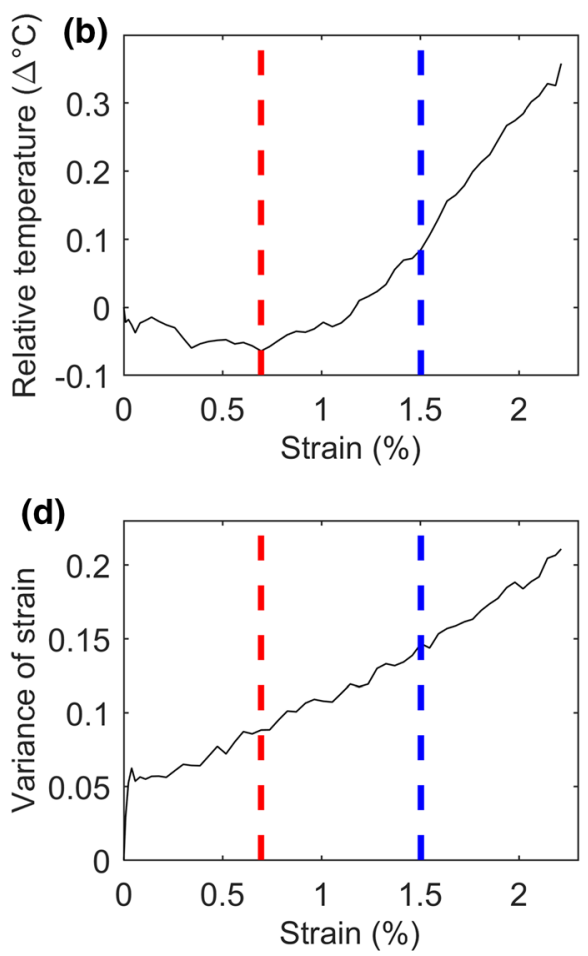

$1.5 \%$ strain shows the onset of damage localisation, where the damage (failure of fiber-fiber bonds) is becoming locally concentrated in certain regions of the paper, which is indicated (c) by an increase in local temperature variance. Strain localisation, the concentration of paper strain in less stiff regions of the paper, starts immediately as indicated by strain variance $(\mathbf{d})$ 
systematic manner a quantitative statistical analysis is utilized.

Linking strain to temperature increase:

understanding damage localization and fracture initiation

Before entering to the statistical analysis of the local properties the development of strain and temperature maps during tensile test is discussed. Fig. 6 shows averaged (all four specimen included) stress, averaged temperature, variance of temperature and variance of strain as a function of paper strain. It can be seen, Fig. $6 \mathrm{~b}$, that the temperature decreased first in the elastic region of the stress-strain curve and then increased considerably after reaching the plastic region. This behavior is well in line with previous studies and can be explained with thermoelastic effect (Dumbleton et al. 1973; Hagman and Nygårds 2017). The increase of the temperature at $0.7 \%$ strain (red line) indicates the onset of fiber-fiber bond failure, which, as discussed above, is caused by the heat dissipation of the strain energy in the fibers when the bonds break. This corroborates with results from acoustic testing (Isaksson and Hägglund 2013) of paper during tensile testing where the onset failure of fiber-fiber bonds was also observed at a strain considerably below the macroscopic failure of the paper.

The variance of temperature describes the localization of damage in the sample, Fig. 6c, i.e. when regions with locally higher damage are forming. The variance of temperature decreased also first in the elastic region. According to Hagman and Nygårds (2017) this can be explained with reduced noise due to cooling of the strip. However, the variance of temperature started to increase only in the middle of the plastic region (blue line at $1.5 \%$ strain). In other words, the damage started to strongly localize after reaching the second half of the plastic region, well after the initiation of bond failure (red line). Interestingly, the variance of strain increased constantly over the whole strain range, Fig. $6 \mathrm{~d}$. This in turn means that the strain started to localize much earlier in comparison to damage. Taking into consideration that local strain and local temperature increase correlate, Fig. 7, it is plausible that the local damage was preceded by the high local strain. This is well in line with the findings of Borodulina et al. (2012) who concluded

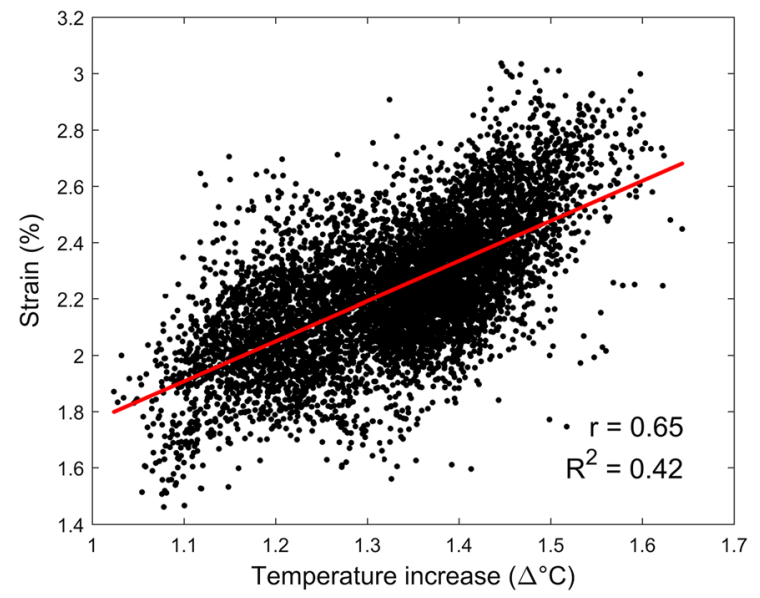

Fig. 7 Scatterplot showing the relationship between local strain and local temperature (all four paper strips included). Each point in the scatterplot corresponds to a $1 \times 1 \mathrm{~mm}^{2}$ spot on a paper specimen and its temperature and local strain, respectively. There is a clear relation between local strain and local failure, i.e. spots with higher local strain also have locally more failing fiber-fiber bonds and hence a higher temperature

that high local strain is the precursor for local failure of fiber-fiber bonds. In other words, the data presented here suggests, that already at a low load regions with locally higher strain are emerging. After onset of bond failure at $0.7 \%$ strain the strain keeps on localizing, the damage initially is not showing increasing inhomogeneity. This is changing at $1.5 \%$ strain where also the damage is starting to localize and regions with locally concentrated bond failure are occurring. Catastrophic failure (sheet rupture) finally initiates within one of the regions where local failure has accumulated and reached a critical level. In low density papers, according to Krasnoshlyk et al. (2018b), the failure propagation in the fracture process zone ahead of the crack tip is seen as an increase in paper thickness and porosity. These increases are caused by rotation and twist of the bonded fibers and gradual breaking of the fiber-fiber bonds. Figure 1b shows the damage (temperature) distribution just prior to rupture of a sheet. The white circle shows a region with significantly increased temperature, local failure has started to heavily concentrate in this region, sheet rupture is about to initiate in this region.

Figure 7 shows a scatterplot describing the pointwise relationship between local temperature increase maps and local strain maps of the four paper strips just prior to rupture, i.e. at the endpoint of the stress-strain 
curve shown in Fig. 6. Each spot in this scatterplot is representing one physical $1 \times 1 \mathrm{~mm}^{2}$ subarea on the sample and the respective temperature and strain values in this spot, like e.g. the green spot at coordinates $x=20 / y=10$ in Fig. 5 e, f. It can be seen that a positive linear correlation exists between these two distributions $(r=0.65)$. By taking into account that the local temperature increase was preceded by the high local strain, it can be said by fitting a linear regression model and calculating a coefficient of determination $\left(R^{2}\right)$ that the local strain explains $42 \%$ of the total variation in the local temperature increase $\left(R^{2}=0.42\right)$. This also means that the remaining difference $58 \%$ are explained by other variables and local strain is not the only indicator for local failure. Hence local strain and the local temperature increase, although related, are partially different which supports the conclusion that local
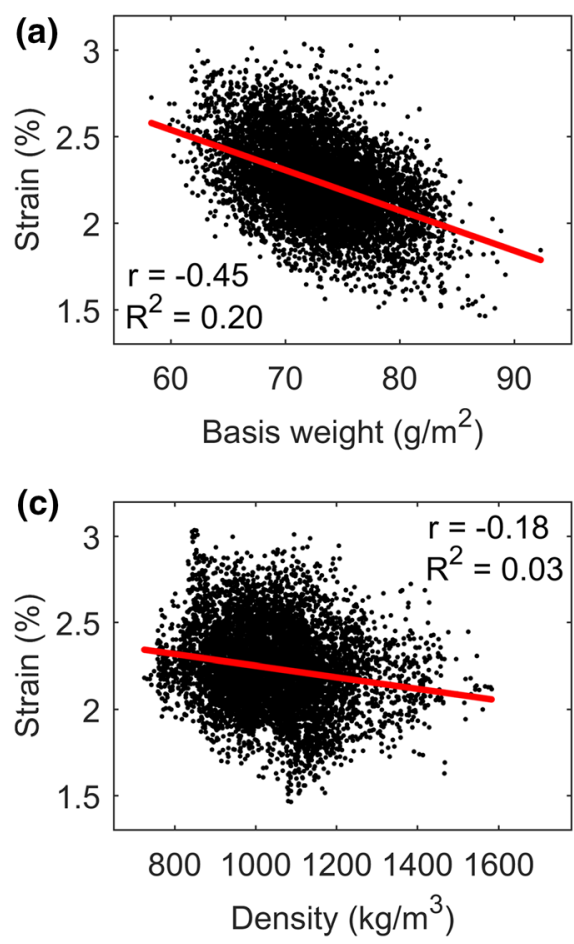

Fig. 8 Relationship between local paper structure and local strain. Each point in a scatterplot corresponds to a $1 \times 1 \mathrm{~mm}^{2}$ spot on a paper specimen and its local properties. Spots with higher basis weight (a) and higher fiber orientation in load direction FOLCF (d) tend to exhibit lower strain during tensile testing, $r=-0.45$ and $r=-0.37$, respectively. A weaker relationship is also found for regions with higher density paper strain and local paper failure are related, but not governed by the exactly same parameters.

In the following the above analyzed local strain and temperature maps are linked to the corresponding paper structure.

Linking local strain and local material failure to paper structure

We begin the analysis by studying the pointwise correlation between strain maps and structural maps of the four paper strips. Figure 8 shows scatterplots describing these relationships. Like described in "Linking procedure" section and illustrated in Fig. 5 each spot in a scatterplot represents one $1 \times 1 \mathrm{~mm}^{2}$ subarea on a paper specimen, and its respective local properties.

First it can be observed that the existing correlations are negative, $r<0$. Local strain of the paper tends
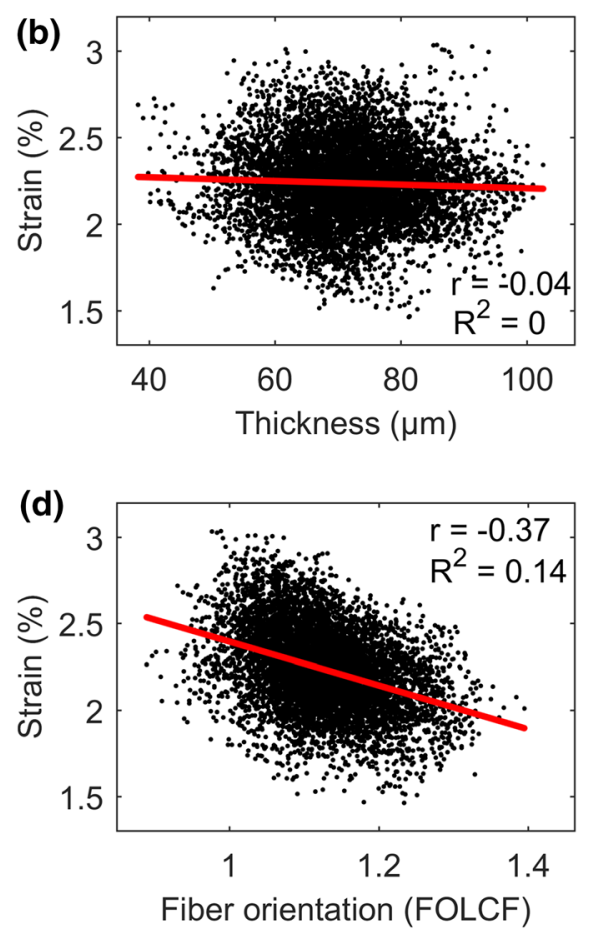

exhibiting lower local strain (c), $r=-0.18$. No correlation exists between local thickness and local strain (b), $r=-0.04$. It can be concluded that local fiber orientation and local grammage variations are the most relevant paper structural properties governing local strain of the material. Less relevant for local strain development is local density of the paper, local thickness variations of the paper are not playing a role 
to be higher for regions with less local grammage, lower local density and less fiber pointing in the direction of the load (FOLCF). The highest, yet still moderate, negative linear correlation exists between local strain and local basis weight $(r=-0.45)$. Furthermore, the local basis weight explains $20 \%$ of the total variation in local strain $\left(R^{2}=0.20\right)$. A relevant negative linear correlation exists also between local strain and local fiber orientation (FOLCF, $r=-0.37$ ). The local FOLCF is capable of explaining $14 \%$ of the total variation in local strain $\left(R^{2}=0.14\right)$, i.e. it is only a slightly weaker predictor for local strain than local basis weight. This is a remarkable result, it suggests that local fiber orientation is about equally relevant for the local strain of paper under tensile load like local grammage. Interestingly enough, Praast and Göttsching (1997) concluded that the local orientation of fibers correlates with the orientation of flocs (high basis weight regions). This means that the orientation of flocs might influence on the local strain as well. A weak negative linear correlation is in turn present between local strain and local density $(r=-0.18)$. The local density explains $3 \%$ of total variation in local strain $\left(R^{2}=0.03\right)$. These results indicate, that the well known relations between the structural properties of paper, i.e. basis weight, fiber orientation and density, and paper stiffness described in "Introduction" section also apply to the local straining under tensile load. Finally, no correlation is present between local strain and local thickness $\left(r=-0.04\right.$ and $\left.R^{2}=0\right)$. This can be explained with the fact that density variation is independent of thickness variation (Dodson et al. 2001).

Dodson (1970) found earlier a significant correlation between local deformation (dilatation) and local basis weight of fibrous networks (see "Introduction" section). Furthermore, he predicted this relation by using a statistical theory of elasticity. In the following we utilize the same approach to predict the relation between local basis weight and local strain shown in Fig. 8a. The theoretical $(t h)$ relative strain $\left(\widetilde{\delta}_{t h}\right)$ as a function of local grammage $(\widetilde{g})$, i.e. theoretical regression line, was locally calculated with the following equation: $\widetilde{\delta}_{t h}=\frac{\widetilde{\varepsilon}_{t h}}{\bar{\varepsilon}}=1+\frac{(1 / \overline{(\bar{g} / \widetilde{g})}-1)}{\operatorname{var}(\widetilde{g})} \bar{g}(\widetilde{g}-\bar{g})$

where strain is denoted by $\varepsilon$, local value by tilde and global mean value by bar. The theoretical correlation coefficient $\left(r_{t h}\right)$ was in turn calculated as follows:

$r_{t h}=\frac{(1 / \overline{(\bar{g} / \widetilde{g})}-1) \bar{g}}{\sqrt{\operatorname{var}(\widetilde{g}) \operatorname{var}(\widetilde{\delta})}}$.

This allowed the theoretical prediction for scatter about the theoretical regression line by calculating the standard deviation $(\sigma)$ of the scatter:

$\sigma_{t h}^{2}=\left(1-r_{t h}^{2}\right) \operatorname{var}(\widetilde{\delta})$.

In Fig. 9 the theoretical regression line as well as $\pm 2 \sigma_{t h}$ theoretical limits for scatter are plotted together with the experimental data (see Fig. 8a). It can be seen that the theoretical predictions are in good agreement with the experimental relations. i.e. the slopes of experimental and theoretical regression lines are close to each other, $95 \%$ of the experimental data points fall within the error bounds and the difference between $r_{t h}=-0.59$ and $r=-0.45$ is not large. These conclusions were also made by Dodson (1970) and his correlation coefficients $r_{t h}=-0.56$ and $r=-0.52$

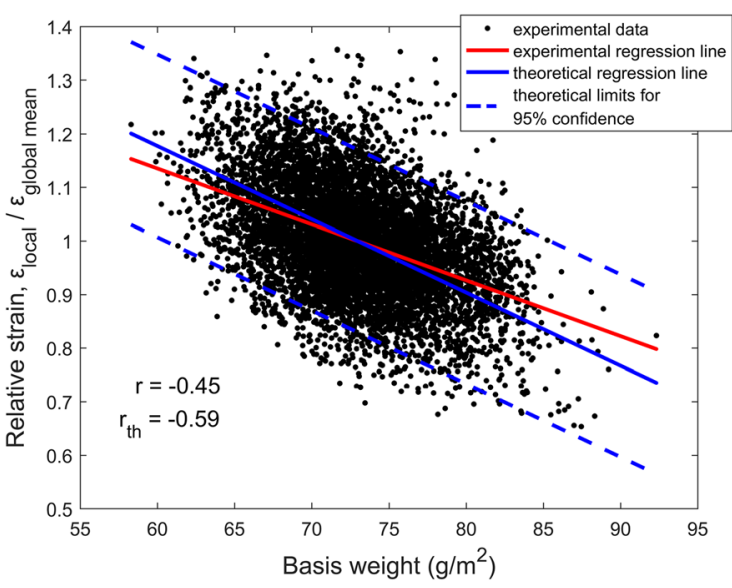

Fig. 9 Prediction of the relation between local relative strain and local basis weight by using a statistical theory of elasticity (Dodson 1970). Each point in a scatterplot corresponds to a $1 \times 1 \mathrm{~mm}^{2}$ spot on a paper specimen and its local relative strain and local basis weight (see Fig. 8a). The theoretical predictions are in good agreement with the experimental relations, and thus the statistical theory of elasticity is suitable for predicting the relation between local strain and local grammage of Kraft paper 

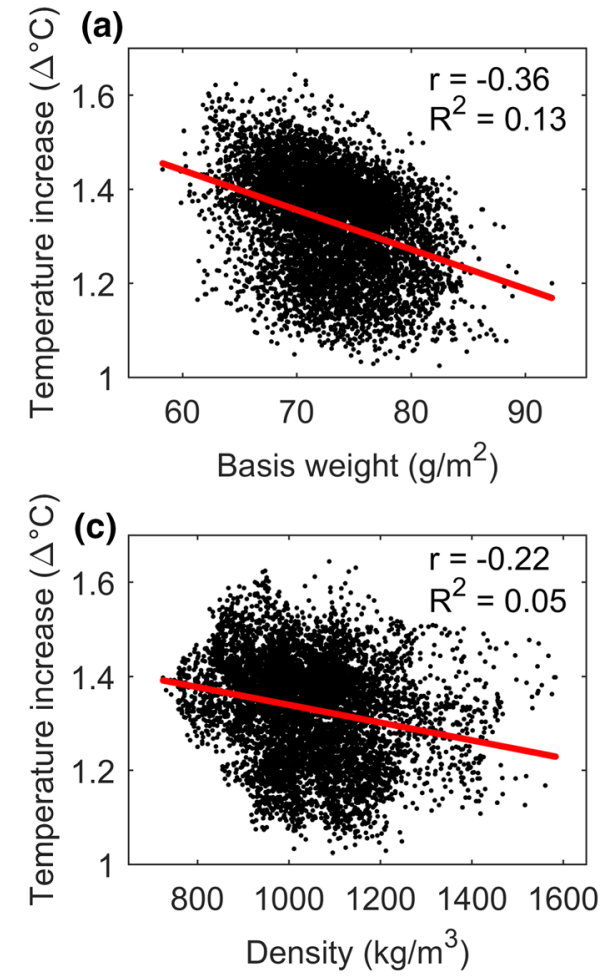

Fig. 10 Relationship between local paper structure and local material damage progression indicated by IR thermograpy. Each point in a scatterplot corresponds to a $1 \times 1 \mathrm{~mm}^{2}$ spot on a paper specimen and its local properties. Regions with less local damage (failure of fiber-fiber bonds) and thus lower temperature tend to have higher local basis weight $\mathbf{a} r=-0.36$, higher

are similar to ours. Thus, the statistical theory of elasticity is suitable for predicting the relation between local strain and local grammage also in the case of Kraft paper.

Scatterplots describing the pointwise relationship between local damage (temperature increase maps) and local structural maps of the four paper strips are in turn shown in Fig. 10. Similar like for the local straining behavior, also local failure of fiber-fiber bonds (temperature increase) tends to occur more pronounced in paper regions with less local grammage, lower local density and less fiber pointing in the direction of the load (FOLCF). The strength of the negative linear correlation between local temperature increase and local basis weight $(r=-0.36)$ is slightly weaker than the correlation between local strain and local basis weight. The local basis weight explains $13 \%$ of the total variation in local temperature increase $\left(R^{2}=0.13\right)$. A weaker negative linear correlation is
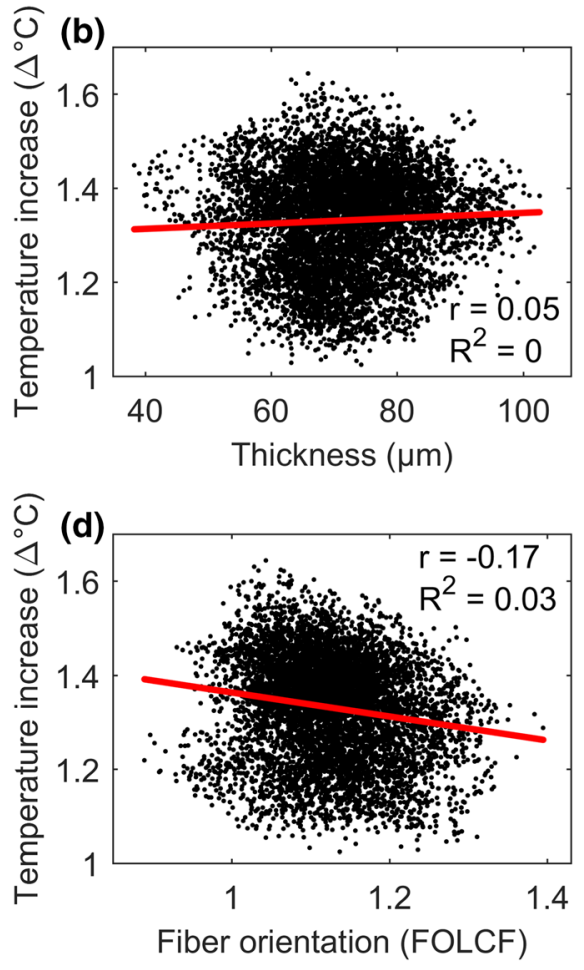

local paper density $\mathbf{c} r=-0.22$, and higher fiber orientation in load direction FOLCF $\mathbf{d} r=-0.17$. It can be concluded that the local failure of fiber-fiber bonds during tensile testing is most influenced by local grammage variations, less relevant seem to be local variations of fiber orientation and paper density

also present between local temperature increase and local fiber orientation (FOLCF, $r=-0.17$ ). The local FOLCF is capable of explaining only $3 \%$ of the total variation in local temperature increase $\left(R^{2}=0.03\right)$. On the other hand, the linear negative correlation is at the same level between local temperature increase and local density $(r=-0.22)$ in comparison to the correlation between local strain and local density. The local density explains $5 \%$ of the total variation in local temperature increase $\left(R^{2}=0.05\right)$. As in the case of local strain and local thickness, no correlation exists between local temperature increase and local thickness $\left(r=0.05\right.$ and $\left.R^{2}=0\right)$. In general the correlations of local paper structure to local bond failure, Fig. 10, are somewhat lower than to local strain, Fig. 8. It is unclear if this difference is significant or not.

So far simple linear regression model has been utilized to study the relationships between local strain, local temperature increase and structural maps. In the 
following the analysis is extended to multiple linear regression model. This means that the local strain and local temperature increase are modelled as a linear function of local basis weight, local thickness, local density and local FOLCF. The analysis revealed that the structural properties combined explain $39 \%$ of the total variation in local strain $\left(R^{2}=0.39\right)$. In other words, the local basis weight, local thickness, local density and local FOLCF are locally able to predict the local strain to a degree of $39 \%$. In the case of local temperature increase the structural properties combined explain in turn $25 \%$ of the total variation. This means that the structural properties predict the local strain better than the local failure of the material (temperature increase). The remaining differences of $R^{2}$ to 1 is 0.61 and 0.75 for the local strain and the local temperature increase, respectively. These differences can be explained with other influence factors, i.e. other structural variables not measured, inaccuracies/noise in the measurements, data map registration errors, et cetera. Analyzing the intercorrelation between the paper structural parameters revealed low linear dependance, which is also confirmed by the significantly higher correlation of the multiple regression model than the individual correlations. This leads to the conclusion that the structural parameters paper formation (local grammage), local fiber orientation and local density are independently responsible for the weak spots contributing to the distribution of local strain and local bond failure in paper.

The final catastrophic failure, i.e. sheet rupture, is always initiating from regions with locally higher strain and bond failure observed already earlier, at lower load. In the final part of the analysis we are focusing on these more extreme regions of the paper, i.e. the regions with particularly high and low local strain and local fiber bond failure. In order to do that the amount of analyzed pixels was reduced as illustrated in Fig. 11. More and more pixels were removed from the middle local strain/temperature increase range and only pixels from the lowest and highest ends were included to the analysis. For example when $10 \%$ of the strain pixels were included, $5 \%$ of the pixels with highest strain and $5 \%$ of the pixels with lowest strain were chosen to the analysis. Pixelwise correlations between local structural properties, local strain and local temperature increase were then investigated similar like above, but utilizing the selected pixels only.

Figure 12 shows that the correlations and $R^{2}$ values (simple linear regression models) between local strain and temperature increase and local structural properties generally increase with decreasing percentage of pixels. This means that the structural paper properties are better able to predict the local strain and local fiber bond failure in the more extreme regions of the paper.

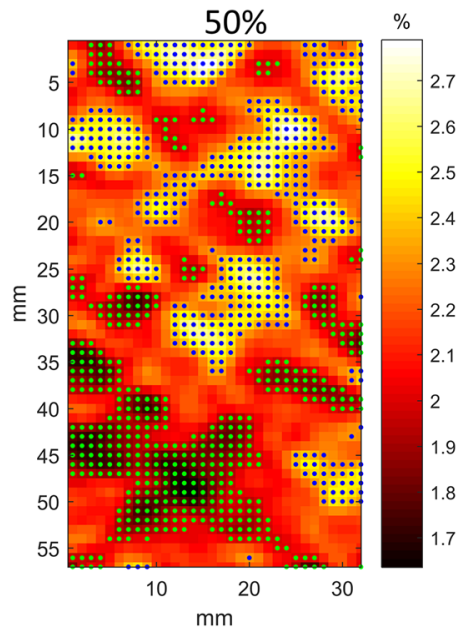

Fig. 11 In order to analyze the properties of regions with extreme properties we are choosing paper areas with particularly high and low strain. We start by analyzing only $50 \%$ of the regions (left), picking the $25 \%$ of regions with the highest strain (blue) and the $25 \%$ of regions with the lowest strain (green).
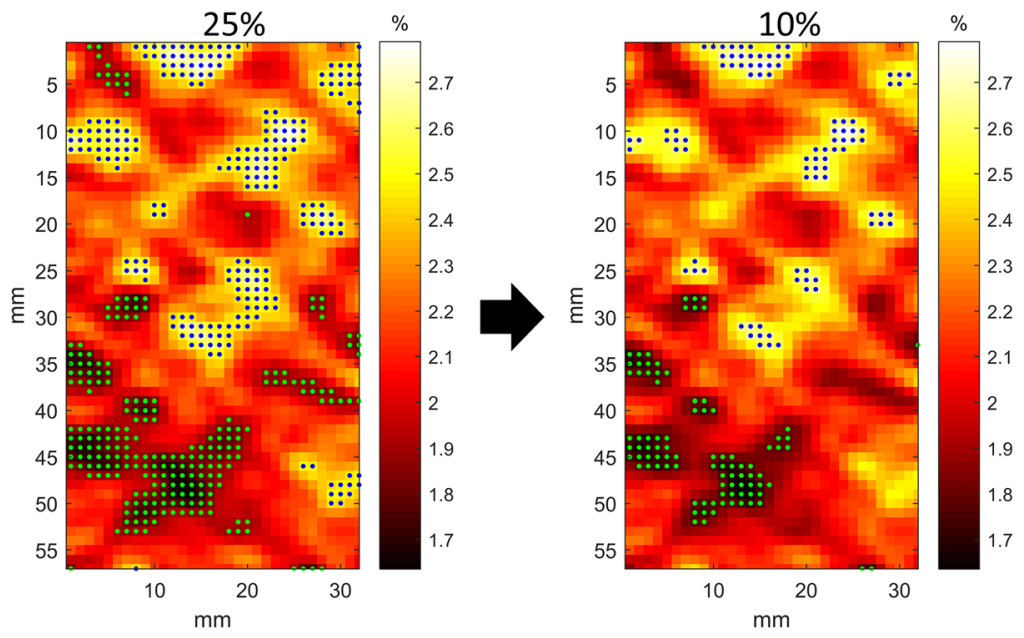

More extreme regions are chosen by picking the $25 \%$ most extreme regions (middle) i.e. $12.5 \%$ of highest and $12.5 \%$ of lowest strain, and the $10 \%$ most extreme regions (right) i.e. $5 \%$ of highest and 5\% of lowest strain. The resulting correlations are shown in Fig. 12 


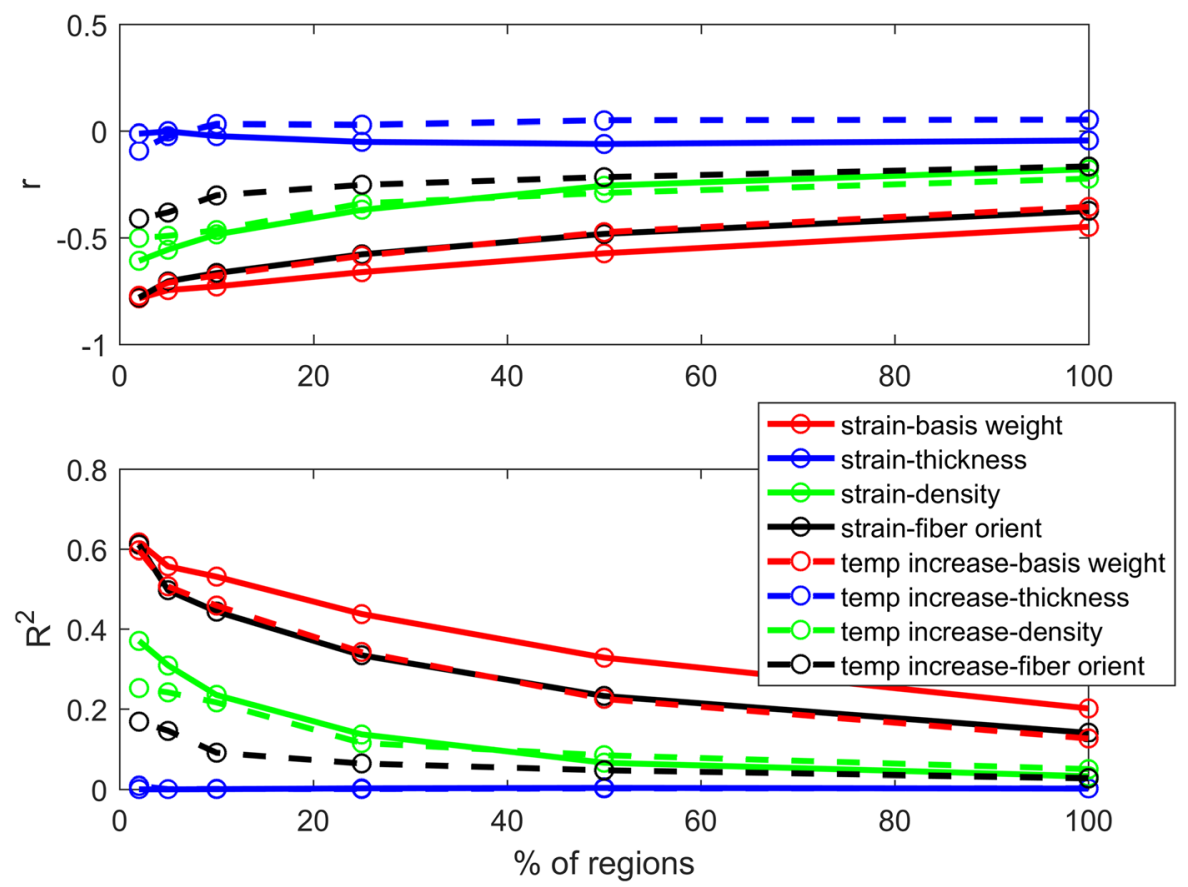

Fig. 12 Relationship ( $r$ and $R^{2}$ ) between local strain/temperature and local structural properties. $100 \%$ means that all regions were used to calculate the correlations like in Figs. 8 and 10 . Going down from $100 \%$ only the pixels with the highest and lowest strain or temperature are used to calculate the correlations, as illustrated in Fig. 11. In this way only the most extreme regions are evaluated as the final sheet rupture is initiating in these highly strained regions. The regions with more

The linear correlation between local strain/temperature increase and local thickness differs from this behavior as no correlation started to occur even at low pixel percentages. This further illustrates the fact that the density variation is independent of thickness variation. It is interesting to note that the best predictors in descending order for the regions with pronounced local strain (solid lines) are: local basis weight, local fiber orientation (FOLCF) and local density (as in Fig. 8 where $100 \%$ of the regions included). However, for prediction of local failure the order (descending) is in turn following: local basis weight, local density and local fiber orientation (FOLCF) (as in Fig. 10 where $100 \%$ of the regions included).

Finally in Fig. 13 the local strain and local temperature increase are modeled as a linear combination of the local structural properties with only more and more extreme regions being considered (as extreme values for strain/temperature (low percentage of regions) show a stronger interrelation with the paper structural properties. The best predictors in descending order for the local strain are: local basis weight, local fiber orientation (FOLCF) and local density. For prediction of local failure the order (descending) is in turn following: local basis weight, local density and local fiber orientation (FOLCF)

explained in Fig. 11). It can be seen from Fig. 13 that the $R^{2}$ value increases with the decreasing pixel amount in both cases. The local structural properties combined explain the total variation in local strain overall better in comparison to the local bond failure (temperature increase) of the paper. In the case of $2 \%$ of pixels the local structural properties combined are locally able to predict the local strain to a degree of $87 \%$ and the local temperature increase to a degree of $72 \%$. The results suggest that for the most extreme regions on the paper (i.e. the regions with the highestand lowest deformation), the local variation of the analyzed paper structural properties local grammage, local fiber orientation and local density is highly related to the local concentrations of paper strain and fiber-fiber bond failure.

The relations in the current study were found by investigating unbleached softwood Kraft paper. However, these relations might differ to various degrees 


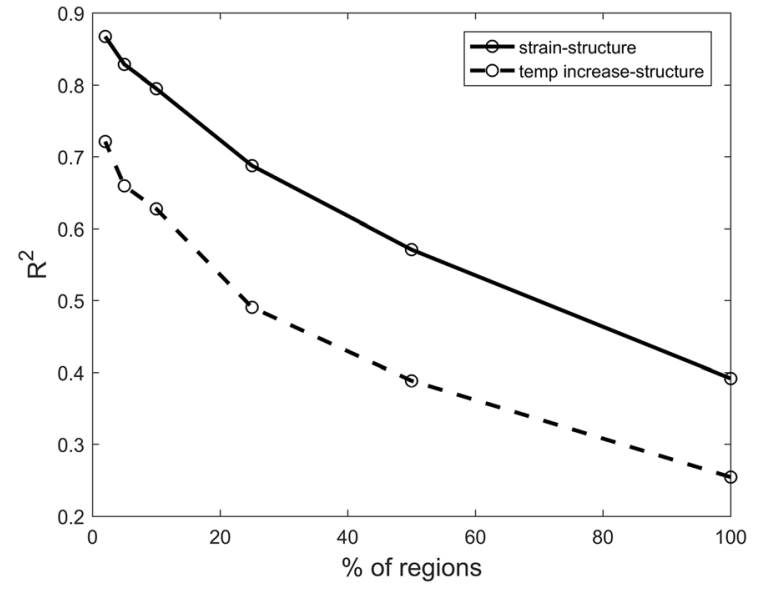

Fig. 13 Correlation coefficient for modeling the local strain and the local damage (temperature increase) as a linear combination of all four local structural parameters (local grammage, fiber orientation, thickness and density). Considering all regions on the paper (100\% of regions) the structural parameters can predict $R^{2}=0.39$ and $R^{2}=0.25$ of the local strain and damage, respectively. For the most extreme regions on the paper with highest and lowest strain and damage the correlation coefficient rises to $R^{2}=0.87$ and $R^{2}=0.72$, respectively

depending on the paper grade. For example, the density of paper (Krasnoshlyk et al. 2018a) and addition of dry-strength agent (Isaksson and Hägglund 2013) influence on the strain and damage distribution in paper. Thus, in the future it would be interesting to investigate how well the findings of the current study apply to other paper grades.

\section{Conclusions}

We have introduced a novel method to quantitatively study the influence of local paper properties (variation of local grammage, local fiber orientation, local density and local thickness) on the development and concentration of local strain and local fiber-fiber bond failure. Using image registration we combined different measurements of local structural properties with DIC measurements of local strain as well as local temperature (as a measure for fiber bond failure due to thermal strain energy dissipation). Applying multiple linear regression on the collected data for $1 \mathrm{~mm}^{2}$ large subregions on the paper samples we quantified the individual and combined influence of different local structural parameters on the localized strain and damage initializing the failure of paper.
We found that strain started to localize much earlier in comparison to fiber bond failure (local temperature increase). Regions with higher strain start to develop even under low load, locally pronounced failure only starts to show at considerably higher load. Pixelwise correlation revealed that the local strain was locally able to predict the local temperature increase to a degree of $42 \%$. Thus we conclude that, although they are related, local strain and local failure of paper are not governed by exactly the same paper structural features.

Analyzing the development of local strain in paper we found that regions with higher basis weight and higher fiber orientation in load direction (FOLCF) tend to exhibit lower strain during tensile testing. Furthermore, the relation between local strain and local grammage can be predicted with the statistical theory of elasticity. Also regions with higher density tend to exhibit lower local strain, but not as pronounced. The findings for local failure of paper are similar, but not equivalent. The strongest correlation exists with local grammage. Local density and local fiber orientation show in turn weaker correlation with local bond failure. Local variations in paper thickness were not relevant in any case.

These findings are broadening the perspective on the structural mechanisms governing paper failure. In the past, failure initiation has been particularly related to paper formation, i.e. local variations of basis weight. The present work provides quantitative evidence that local fiber orientation and local density variations are equally relevant as paper formation (local grammage variations) in creating weak spots in paper, and thus in causing local concentrations of paper strain and local initiation of paper failure. In the future it would be interesting to investigate how well these findings apply to different paper grades.

Acknowledgments The financial support by the Austrian Federal Ministry for Digital and Economic Affairs and the National Foundation for Research Technology and Development is gratefully acknowledged. Furthermore, the authors want to thank Mondi, Canon Production Printing, Kelheim Fibres and SIG Combibloc for their financial support.

Funding Open access funding provided by Graz University of Technology. Funding was provided by Christian Doppler Forschungsgesellschaft (Grant: CD Laboratory for Fiber Swelling and Paper Performance).

Data availability The relevant DIC hard- and software parameters are given in Online Resource 1. 


\section{Compliance with ethical standards}

Conflict of interest The authors declare that they have no conflict of interest.

\section{Code availability Not applicable.}

Open Access This article is licensed under a Creative Commons Attribution 4.0 International License, which permits use, sharing, adaptation, distribution and reproduction in any medium or format, as long as you give appropriate credit to the original author(s) and the source, provide a link to the Creative Commons licence, and indicate if changes were made. The images or other third party material in this article are included in the article's Creative Commons licence, unless indicated otherwise in a credit line to the material. If material is not included in the article's Creative Commons licence and your intended use is not permitted by statutory regulation or exceeds the permitted use, you will need to obtain permission directly from the copyright holder. To view a copy of this licence, visit http://creativecommons.org/licenses/by/4.0/.

\section{References}

Alava M, Niskanen K (2008) In-plane tensile properties. In: Papermaking science and technology, vol 16, 2nd edn, Chap 5. Finnish Paper Engineers' Association, Helsinki, pp 181-228

Battams GP, Dulieu-Barton JM (2016) Data-rich characterisation of damage propagation in composite materials. Compos Part A Appl Sci Manuf 91:420-435. https://doi.org/10. 1016/j.compositesa.2016.08.007

Borodulina S, Kulachenko A, Galland S, Nygårds M (2012) Stress-strain curve of paper revisited. Nord Pulp Pap Res J 27(2):318-328. https://doi.org/10.3183/npprj-2012-27-02p318-328

Dodson CTJ (1970) The statistical analysis of patterns of deformation in flat bonded fibrous networks. J Phys D Appl Phys 3(3):269-276. https://doi.org/10.1088/0022-3727/3/ $3 / 305$

Dodson CTJ, Oba Y, Sampson WW (2001) On the distribution of mass, thickness and density in paper. Appita J 54(4):385-389

Dumbleton DP, Kringstad KP, Soremark C (1973) Temperature profiles in paper during straining. Svensk Papperstidning 76(14):521-528

Eberl C, Thompson R, Gianola D, Sharpe Jr W, Hemker K (2006) Digital image correlation and tracking. In: MatLabCentral, mathworks file exchange server. FileID 12413. Accessed 4 April 2017

Goidescu C, Welemane H, Garnier C, Fazzini M, Brault R, Péronnet E, Mistou S (2013) Damage investigation in cfrp composites using full-field measurement techniques: combination of digital image stereo-correlation, infrared thermography and $\mathrm{x}$-ray tomography. Compos Part B Eng 48:95-105. https://doi.org/10.1016/j.compositesb.2012. 11.016
Hagman A, Nygårds M (2012) Investigation of sample-size effects on in-plane tensile testing of paperboard. Nord Pulp Pap Res J 27(2):295-304. https://doi.org/10.3183/npprj2012-27-02-p295-304

Hagman A, Nygårds M (2017) Thermographical analysis of paper during tensile testing and comparison to digital image correlation. Exp Mech 57(2):325-339. https://doi. org/10.1007/s11340-016-0240-4

Hirn U, Bauer W (2007) Evaluating an improved method to determine layered fiber orientation by sheet splitting. In: 61st Annual APPITA conference and 2007 paper physics conference, pp 71-80

Hirn U, Lechthaler M, Bauer W (2008) Registration and point wise correlation of local paper properties. Nord Pulp Pap Res J 23(4):374-381. https://doi.org/10.3183/npprj-200823-04-p374-381

Hyll C (2012) Infrared emittance of paper: method development, measurement and application. Licentiate thesis, The Royal Institute of Technology, Stockholm

I'Anson SJ, Sampson WW, Savani S (2008) Density dependent influence of grammage on tensile properties of handsheets. J Pulp Pap Sci 34(3):182

Isaksson P, Hägglund R (2013) Acoustic emission assisted fracture zone analysis of cellulose fibre materials. J Compos Mater 47(22):2865-2874. https://doi.org/10.1177/ 0021998312459781

Keller DS, Pawlak JJ (2001) $\beta$-radiographic imaging of paper formation using storage phosphor screens. J Pulp Pap Sci 27(4):117-123

Keller DS, Branca DL, Kwon O (2012) Characterization of nonwoven structures by spatial partitioning of local thickness and mass density. J Mater Sci 47(1):208-226. https://doi.org/10.1007/s10853-011-5788-x

Korteoja MJ, Lukkarinen A, Kaski K, Gunderson DE, Dahlke JL, Niskanen KJ (1996) Local strain fields in paper. Tappi J 79(4):217-224

Korteoja MJ, Lukkarinen A, Kaski K, Niskanen KJ (1997) Computational study of formation effects on paper strength. J Pulp Pap Sci 23(1):J18-J22

Korteoja MJ, Niskanen KJ, Kortschot MT, Kaski KK (1998) Progressive damage in paper. Pap Puu 80(5):364-372

Krasnoshlyk V, Rolland du Roscoat S, Dumont PJJ, Isaksson P (2018a) Influence of the local mass density variation on the fracture behavior of fiber network materials. Int J Solids Struct 138:236-244. https://doi.org/10.1016/j.ijsolstr. 2018.01.016

Krasnoshlyk V, Rolland du Roscoat S, Dumont PJJ, Isaksson P, Ando E, Bonnin A (2018b) Three-dimensional visualization and quantification of the fracture mechanisms in sparse fibre networks using multiscale $\mathrm{x}$-ray microtomography. Proc R Soc A 474:20180175. https://doi.org/10.1098/rspa. 2018.0175

Neter J, Kutner MH, Nachtsheim CJ, Wasserman W (1996) Applied linear statistical models, 4th edn. McGraw-Hill, New York

Niskanen K, Pakarinen P (2008) Paper structure. In: Papermaking Science and Technology, vol 16, 2nd edn, Chap. 1. Finnish Paper Engineers' Association, Helsinki, pp 11-58

Norman RJ (1965) Dependence of sheet properties on formation and forming variables. In: Bolam F (eds) Consolidation of 
the Paper Web, Trans IIIrd Fund Res Symp Cambridge, vol 1. FRC, Manchester, pp 269-298

Pan B, Qian K, Xie H, Asundi A (2009) Two-dimensional digital image correlation for in-plane displacement and strain measurement: a review. Meas Sci Technol 20(6):062001. https://doi.org/10.1088/0957-0233/20/6/ 062001

Praast H, Göttsching L (1997) Local orientation of flocs in paper. In: Baker CF (eds) The fundamentals of papermaking materials, Trans XIth Fund Res Symp Cambridge, vol 2. FRC, Manchester, pp 1293-1324

Romanova AN, Kazakov Y, Malkov A (2020) Quantitative characteristics of local strain fields in a top-liner board sample under uniaxial tension. Lesnoy Zhurnal (Russ For J) 1:180-189. https://doi.org/10.37482/0536-1036-2020-1180-189
Wong L, Kortschot MT, Dodson CTJ (1996) Effect of formation on local strain fields and fracture of paper. J Pulp Pap Sci 22(6):J213-J219

Yamauchi T (2012) Application of IR thermography for studying deformation and fracture of paper. In: INTECH open access publisher, Chap 1. Infrared Thermography, Rijeka, pp 3-26. https://doi.org/10.5772/29961

Yamauchi T, Murakami K (1994) Observation of deforming process of a poorly formed papersheet by thermography. Sen-i Gakkaishi 50(9):424-425

Publisher's Note Springer Nature remains neutral with regard to jurisdictional claims in published maps and institutional affiliations. 